\title{
Signature of Gravitational Waves in Stellar Spectroscopy
}

\author{
Shahen Hacyan \\ Instituto de Física, Universidad Nacional Autónoma de México, Ciudad de México, Mexico \\ Email: hacyan@fisica.unam.mx
}

Received 16 January 2016; accepted 27 March 2016; published 31 March 2016

Copyright (C) 2016 by author and Scientific Research Publishing Inc.

This work is licensed under the Creative Commons Attribution International License (CC BY).

http://creativecommons.org/licenses/by/4.0/

(c) (i) Open Access

\begin{abstract}
The possible detection of gravitational waves by interferometric observations of distant light sources is studied. It is shown that a gravitational wave affects the interferometric pattern of stellar light in a particular way. Michelson and Hanbury Brown-Twiss interferometers are considered, and it is shown that the latter is the most adequate for such a detection.
\end{abstract}

\section{Keywords}

\section{Gravitational Waves, Spectroscopy}

\section{Introduction}

A gravitational wave (GW) could be detected indirectly by its interaction with the light emitted by astronomical objects. Thus, for instance, the passage of a GW produces a time delay in the signal received from distant sources (Estabrook and Wahlquist [1]). Similarly, the presence of a stochastic background of GWs can be inferred from a statistical analysis of pulsar timing (Hellings and Downs [2]). GWs can also interact with the polarization of electromagnetic waves (Hacyan [3] [4]).

In this paper, we study the effect of GWs on the interferometry of stellar light. Two basic types of interferometric devices used in astronomy are considered: the Michelson (see, e.g., [5]) and the Hanbury Brown-Twiss [6] interferometers. The former uses the interference between two signals, and the latter uses the interference between intensities of light. An intensity interferometer has, in general, some advantages over a Michelson interferometer. It will be shown in the following that the passage of a GW could be more easily detected by intensity interferometry.

Section 2 of the present paper is devoted to the analysis of an electromagnetic wave in the presence of a plane fronted GW. The analysis is based on previous works (Hacyan [3] [4]) in which the form of the electromagnetic 
field is deduced using a short-wave length approximation. A general formula for the correlation of electric fields is obtained and the result is applied to interferometric analysis in Section 3; particular cases are worked out.

\section{The Electromagnetic Field}

The metric of a plane GW in the weak field limit is

$$
\mathrm{d} s^{2}=-2 \mathrm{~d} u \mathrm{~d} v+(1+f) \mathrm{d} x^{2}+(1-f) \mathrm{d} y^{2}+2 g \mathrm{~d} x \mathrm{~d} y,
$$

where the two degrees of polarization of the GW are given by the potentials $f(u)$ and $g(u)$, which are functions of $u$ only. The relation with Minkowski coordinates $t$ and $z$ is

$$
u=\frac{1}{\sqrt{2}}(t-z), \quad v=\frac{1}{\sqrt{2}}(t+z) .
$$

In the following, quadratic and higher order terms in $f$ and $g$ are neglected, and we set $c=1$.

The direction of a light ray in the absence of a GW is $k$, with $|k|=\omega$, the frequency of the (monochromatic) wave. We set

$$
k=\omega(\sin \theta \cos \phi, \sin \theta \sin \phi, \cos \theta),
$$

thus defining the angles $\theta$ and $\phi$. In the following, it will be convenient to define the functions

$$
\begin{aligned}
& F(u ; \phi)=f(u) \cos 2 \phi+g(u) \sin 2 \phi, \\
& G(u ; \phi)=-f(u) \sin 2 \phi+g(u) \cos 2 \phi .
\end{aligned}
$$

In the short-wave length approximation, the electromagnetic potential is taken as

$$
A_{\mu}=a_{\mu} \mathrm{e}^{i S},
$$

where $S$ is the eikonal function satisfying the equation $g^{\mu v} S_{, \mu} S_{, v}=0$. Then, $K_{\alpha} \equiv S_{, \alpha}$ is a null-vector defining the direction of propagation of the electromagnetic wave, and $a_{\alpha}$ is a four-vector such that $a_{\mu} K^{\mu}=0$.

The electromagnetic vector is [4]

$$
E_{\alpha}=i\left[\Omega a_{\alpha}+\left(a_{\beta} t^{\beta}\right) K_{\alpha}\right] \mathrm{e}^{i S},
$$

where $t^{\alpha}$ is a time-like four-vector and $\Omega=-K_{\mu} t^{\mu}$ is the frequency measured by a detector with $t^{\alpha}$ tangent to its world-line. Choosing $t^{\alpha}=(1 / \sqrt{2})(1,1,0,0)$, it follows that

$$
\Omega(u ; \theta, \phi)=\omega\left[1-\frac{1}{2}(1+\cos \theta) F(u ; \phi)\right],
$$

and the eikonal function is

$$
S(x ; k)=x \cdot k+\frac{\omega}{\sqrt{2}}(1+\cos \theta) \int F\left(u^{\prime} ; \phi\right) \mathrm{d} u^{\prime} .
$$

As in Ref. [4], for a plane wave we use a gauge such that $a_{v}=0$, which is equivalent to

$$
\left(\boldsymbol{n}_{g w}-\omega^{-1} \boldsymbol{k}\right) \cdot \boldsymbol{a}=0,
$$

where $\boldsymbol{n}_{g w}$ is the unit vector in the direction of propagation of the GW.

The four vector $a_{\alpha}$ depends on the coordinate $u$ through the functions $f(u)$ and $g(u)$. With the gauge $a_{v}=0$, a particular solution is [4] 


$$
\begin{aligned}
& a_{u}=\frac{1}{k_{v}}\left[k^{x} a_{x}+k^{y} a_{y}\right] \\
& a_{v}=0 \\
& a_{x}=\left(1+\frac{1}{2} f(u)\right) \bar{a}_{x}+\frac{1}{2} g(u) \bar{a}_{y} \\
& a_{y}=\frac{1}{2} g(u) \bar{a}_{x}+\left(1-\frac{1}{2} f(u)\right) \bar{a}_{y}
\end{aligned}
$$

where $\bar{a}_{x}$ and $\bar{a}_{y}$ are constants defining an electromagnetic plane wave in the absence of GWs.

Let us use a tetrad $e_{(a)}^{\alpha}$ such that $e_{(a)}^{\alpha} e_{(b)}^{\beta} g_{\alpha \beta}=\eta_{(a b)}$, where $\eta_{(a b)}=\operatorname{diag}(1,1,1,-1)$ is the Minkowski matrix. Then, if $\partial_{(a)}=e_{(a)}^{\alpha} \partial_{\alpha}$, the tetrad is defined by

$$
\begin{aligned}
& \partial_{(1)}=\left(1-\frac{1}{2} f(u)\right) \partial_{x}-\frac{1}{2} g(u) \partial_{y} \\
& \partial_{(2)}=-\frac{1}{2} g(u) \partial_{x}+\left(1+\frac{1}{2} f(u)\right) \partial_{y} \\
& \partial_{(3)}=\frac{1}{\sqrt{2}}\left(-\partial_{u}+\partial_{v}\right) \\
& \partial_{(4)}=\frac{1}{\sqrt{2}}\left(\partial_{u}+\partial_{v}\right) .
\end{aligned}
$$

Accordingly the tetrad components of $a_{\alpha}$ and $K_{\alpha}$ are

$$
a_{(n)}=\left(\bar{a}_{x}, \bar{a}_{y},-a_{(4)}, a_{(4)}\right),
$$

and

$$
K_{(n)}=\left(\begin{array}{c}
\left(1-\frac{1}{2} f(u)\right) k_{x}-\frac{1}{2} g(u) k_{y} \\
-\frac{1}{2} g(u) k_{x}+\left(1+\frac{1}{2} f(u)\right) k_{y} \\
\frac{1}{\sqrt{2}}\left[-k_{u}(1-F(u))+k_{v}\right] \\
\frac{1}{\sqrt{2}}\left[k_{u}(1-F(u))+k_{v}\right]
\end{array}\right) .
$$

Notice in particular that $K_{(4)}=-\Omega$, and $\eta^{(m n)} K_{(m)} K_{(n)}=0$, as it should be.

The electric field in tetrad components is

$$
E_{(n)}=i\left(-K_{(4)} a_{(n)}+a_{(4)} K_{(n)}\right) \mathrm{e}^{i s},
$$

and of course $E_{(4)}=0$.

\section{Correlations}

For an electromagnetic plane wave with wave vector $K_{\alpha}(u)$, we find after some lengthy but straightforward algebra (keeping only terms of first order)

$$
\begin{aligned}
\eta^{(m n)} E_{(m)}^{*}(x ; k) E_{(n)}\left(x^{\prime} ; k\right)= & \left\{S_{0}\left[1-\frac{1}{2}(1+\cos \theta)\left(F(u ; \phi)+F\left(u^{\prime} ; \phi\right)\right)\right]\right. \\
& \left.+i S_{3}(1+\cos \theta)\left(G(u ; \phi)-G\left(u^{\prime} ; \phi\right)\right)\right\} \mathrm{e}^{i\left(S\left(x^{\prime} ; k\right)-S(x ; k)\right)},
\end{aligned}
$$


where

$$
\begin{aligned}
& S_{0}=\omega^{2}\left(\left|\bar{a}_{x}\right|^{2}+\left|\bar{a}_{y}\right|^{2}\right), \\
& i S_{3}=\omega^{2}\left(\bar{a}_{y}^{*} \bar{a}_{x}-\bar{a}_{x}^{*} \bar{a}_{y}\right)
\end{aligned}
$$

are Stokes parameters ( $S_{3}=0$ for linear and $\left|S_{3}\right|=S_{0}$ for circular polarizations).

\section{Interferometry}

Consider two detectors with space-time coordinates $x_{1}$ and $x_{2}$, each receiving two plane electromagnetic waves with wave-vectors $k_{1}$ and $k_{2}$, and use the shorthand notation

$$
\begin{aligned}
& \eta^{(m n)} E_{(m)}^{*}\left(x_{a} ; k_{j}\right) E_{(n)}\left(x_{b} ; k_{j}\right) \\
& \equiv E_{a ; j}^{\dagger} E_{b ; j} \equiv S_{0} \exp \left\{\Re_{a j}+i \mathfrak{I}_{a j}+\Re_{b j}-i \mathfrak{I}_{b j}\right\},
\end{aligned}
$$

where

$$
\begin{gathered}
\mathfrak{R}_{a j}=-\frac{1}{2}\left(1+\cos \theta_{j}\right) F\left(u_{a} ; \phi_{j}\right), \\
\mathfrak{J}_{a j}=-S\left(x_{a} ; k_{j}\right)+\frac{S_{3}}{S_{0}}\left(\left(1+\cos \theta_{j}\right) G\left(u_{a} ; \phi_{j}\right),\right.
\end{gathered}
$$

the subindexes $a, b$ and $j$ refer to the labels 1 and 2 of $x$ and $k$.

A Michelson interferometer permits to measure the average intensity

$$
\begin{aligned}
\langle I\rangle \equiv & \left\langle E_{1 ; 1}^{\dagger} E_{1 ; 1}+E_{1 ; 2}^{\dagger} E_{1 ; 2}+E_{2 ; 1}^{\dagger} E_{2 ; 1}+E_{2 ; 2}^{\dagger} E_{2 ; 2}\right\rangle \\
& +\left\langle E_{1 ; 1}^{\dagger} E_{2 ; 1}+E_{2 ; 1}^{\dagger} E_{1 ; 1}+E_{1 ; 2}^{\dagger} E_{2 ; 2}+E_{2 ; 2}^{\dagger} E_{1 ; 2}\right\rangle,
\end{aligned}
$$

where the second term is the interference term.

A Hanbury Brown-Twiss interferometer permits to measure the interference between intensities:

$$
\begin{aligned}
\left\langle I_{1} I_{2}\right\rangle \equiv & \left\langle\left(E_{1 ; 1}^{\dagger} E_{1 ; 1}+E_{1 ; 2}^{\dagger} E_{1 ; 2}\right)\left(E_{2 ; 1}^{\dagger} E_{2 ; 1}+E_{2 ; 2}^{\dagger} E_{2 ; 2}\right)\right\rangle \\
& +\left\langle E_{1 ; 1}^{\dagger} E_{1 ; 2} E_{2 ; 2}^{\dagger} E_{2 ; 1}+E_{1 ; 2}^{\dagger} E_{1 ; 1} E_{2 ; 1}^{\dagger} E_{2 ; 2}\right\rangle,
\end{aligned}
$$

where the second term is the interference between the two intensities.

Define

$$
\begin{aligned}
\mathfrak{R}_{ \pm} & =\mathfrak{R}_{11} \pm \mathfrak{R}_{12}+\mathfrak{R}_{21} \pm \mathfrak{R}_{22} \\
\mathfrak{J}_{ \pm} & =\mathfrak{I}_{11}-\mathfrak{I}_{21} \pm \mathfrak{J}_{12} \mp \mathfrak{J}_{22} .
\end{aligned}
$$

With this notation, we have for a Michelson interferometer:

$$
\langle I\rangle=2 S_{0}\left[\left(2+\mathfrak{R}_{+}\right)\left(1+\cos \left(\mathfrak{I}_{+} / 2\right) \cos \left(\mathfrak{I}_{-} / 2\right)\right)-\mathfrak{R}_{-} \sin \left(\mathfrak{I}_{+} / 2\right) \sin \left(\mathfrak{I}_{-} / 2\right)\right],
$$

and for a Hanbury Brown-Twiss interferometer:

$$
\left\langle I_{1} I_{2}\right\rangle=2 S_{0}^{2}\left(1+\Re_{+}\right)\left(2+\cos \mathfrak{I}_{-}\right) .
$$

Define also the complex functions

$$
h(u)=f(u)+i g(u)
$$

and

$$
\Theta_{i}=\mathrm{e}^{-2 i \phi_{i}} \cos ^{2} \frac{\theta_{i}}{2}
$$


Then

$$
\mathfrak{R}_{ \pm}=-\mathfrak{R} e\left\{\left[h\left(u_{1}\right)+h\left(u_{2}\right)\right]\left(\Theta_{1} \pm \Theta_{2}\right)\right\},
$$

and

$$
\mathfrak{J}_{ \pm}=\left(x_{2}-x_{1}\right) \cdot\left(k_{1} \pm k_{2}\right)+\mathfrak{R} e\left\{\left[\sqrt{2} \omega \int_{u_{1}}^{u_{2}} h\left(u^{\prime}\right) \mathrm{d} u^{\prime}-2 i \frac{S_{3}}{S_{0}}\left(h\left(u_{1}\right)-h\left(u_{2}\right)\right)\right]\left(\Theta_{1} \pm \Theta_{2}\right)\right\} .
$$

In the absence of GWs, $\mathfrak{R}_{ \pm}=0$, and

$$
\begin{gathered}
\mathfrak{J}_{+} \rightarrow-2 \omega\left(t_{2}-t_{1}\right)+\left(\boldsymbol{r}_{2}-\boldsymbol{r}_{1}\right) \cdot\left(\boldsymbol{k}_{1}+\boldsymbol{k}_{2}\right) \\
\mathfrak{I}_{-} \rightarrow\left(\boldsymbol{r}_{2}-\boldsymbol{r}_{1}\right) \cdot\left(\boldsymbol{k}_{1}-\boldsymbol{k}_{2}\right),
\end{gathered}
$$

implying that $\left\langle I_{1} I_{2}\right\rangle$ is time independent. It thus follows that the time variation of $\left\langle I_{1} I_{2}\right\rangle$ is due entirely to the presence of a GW. This time dependence can be made explicit setting

$$
\begin{gathered}
\mathfrak{I}_{+}=-2 \omega \Delta t+\Delta \boldsymbol{r} \cdot\left(\boldsymbol{k}_{1}+\boldsymbol{k}_{2}\right)+\Delta \mathfrak{I}_{+} \\
\mathfrak{I}_{-}=-\Delta \boldsymbol{r} \cdot \Delta \boldsymbol{k}+\Delta \mathfrak{I}_{-}
\end{gathered}
$$

where $\Delta t=t_{2}-t_{1}, \Delta r=\boldsymbol{r}_{2}-\boldsymbol{r}_{1}, \Delta \boldsymbol{k}=\boldsymbol{k}_{2}-\boldsymbol{k}_{1}$, and $\Delta \mathfrak{I}_{ \pm}$are small terms due to the GW. This implies that the terms $\mathfrak{R}_{ \pm}$and $\Delta \mathfrak{I}_{ \pm}$are of first order in the potentials $f$ and $g$ of the GW.

It should be noticed that the field correlation $\langle I\rangle$ contains terms such as $\cos \left[\Delta \boldsymbol{x} \cdot\left(\boldsymbol{k}_{1}+\boldsymbol{k}_{2}\right)\right]$, which are highly oscillatory and hinder a precise measurement with a Michelson interferometer. On the other hand, such terms do not appear in the correlation of the intensities:

$$
\left\langle I_{1} I_{2}\right\rangle=2 S_{0}^{2}\left[\left(1+\Re_{+}\right)[2+\cos (\Delta \boldsymbol{x} \cdot \Delta \boldsymbol{k})]+\Delta \mathfrak{I}_{-} \sin (\Delta \boldsymbol{x} \cdot \Delta \boldsymbol{k})\right] .
$$

The time dependence is included only in the terms $\mathfrak{R}_{+}$and $\Delta \mathfrak{I}_{-}$, which are entirely due to the passage of the GW. The term with $\Delta x \cdot\left(k_{1}+k_{2}\right)$ is not present in this last formula.

\subsection{Temporal Coherence}

As a particular application of the above formulas, we can calculate the temporal coherence of a single signal in the presence of a GW. This can be obtained setting $x_{1}=(t, r), x_{2}=(t+\tau, r)$, and $k_{1}=k_{2} \equiv k$. Then $\mathfrak{R}_{-}=0=\mathfrak{I}_{-}$ and accordingly

$$
\langle I\rangle=2 S_{0}\left(2+\mathfrak{R}_{+}\right)\left(1+\cos \left(\mathfrak{I}_{+} / 2\right)\right)
$$

and

$$
\left\langle I_{1} I_{2}\right\rangle=4 S_{0}^{2}\left(1+\Re_{+}\right) .
$$

Explicitly, in this particular case,

$$
\mathfrak{R}_{+}(t, \tau)=-2 \mathfrak{R} e\{[h(t+\tau)+h(t)] \Theta\},
$$

which is the only relevant term for the time correlation of the intensity correlation, and is entirely due to the GW.

\subsection{Sinusoidal Waves and Pulses}

In the particular case of a sinusoidal monochromatic GW of frequency $\omega_{g w}$, we can set

$$
h(u)=h_{0} \mathrm{e}^{i \omega_{g w} u},
$$

where $h_{0} \equiv\left|h_{0}\right| \mathrm{e}^{i \alpha}$ is a complex constant and $\alpha$ a constant phase. 
As for a pulse of GW, it can be approximated by a delta function: $h(u)=h_{0} \delta\left(u-u_{0}\right)$. In this case, only $\mathfrak{I}_{ \pm}$ is changed after $u>u_{0}$. We have

$$
\mathfrak{I}_{ \pm}\left(u>u_{0}\right)=\mathfrak{I}_{ \pm}\left(u<u_{0}\right)+\sqrt{2} \omega \Re e\left\{h_{0}\left(\Theta_{1} \pm \Theta_{2}\right)\right\} w\left(u_{0} ; u_{1}, u_{2}\right),
$$

where $w\left(u_{0} ; u_{1}, u_{2}\right)$ is a function such that $w=1$ if $u_{1}<u_{0}<u_{2}$ and $w=0$ otherwise. Thus, a pulse of gravitational wave would produce a change both in $\langle I\rangle$ and $\left\langle I_{1} I_{2}\right\rangle$.

\section{Conclusion}

The main conclusion from the present results is that the passage of a GW produces a time-dependent perturbation in the intensity interference of a distant light sources, an interference which would otherwise have a static pattern. Thus, a time variation of $\left\langle I_{1} I_{2}\right\rangle$ will denote the passage of a gravitational wave. A similar effect would be more difficult to observe with $\langle I\rangle$, a direct signal interferometer, due to the presence of highly oscillating terms, as shown above.

\section{References}

[1] Estabrook, G.S. and Wahlquist, H.D. (1975) General Relativity and Gravitation, 6, 439-447. http://dx.doi.org/10.1007/BF00762449

[2] Hellings, R.W. and Downs, G.S. (1983) The Astrophysical Journal, 265, L39-L42. http://dx.doi.org/10.1086/183954

[3] Hacyan, S. (2012) General Relativity and Gravitation, 44, 2923-2931. http://dx.doi.org/10.1007/s10714-012-1434-4

[4] Hacyan, S. (2016) International Journal of Modern Physics A, 31, 1641023, 8 p. http://dx.doi.org/10.1142/S0217751X16410232

[5] Hariharan, P. (2007) Basics of Interferometry. 2nd Edition, Elsevier, Amsterdam.

[6] Hanbury Brown, R. and Twiss, R.Q. (1956) Nature, 178, 1046-1048. http://dx.doi.org/10.1038/1781046a0 